\title{
APC: A new code for Atmospheric Polarization Computations
}

\author{
Sergey V. Korkin ${ }^{\mathrm{a}, \mathrm{b}, *}$, Alexei I. Lyapustin ${ }^{\mathrm{b}}$, Vladimir V. Rozanov ${ }^{\mathrm{c}}$ \\ ${ }^{a}$ Universities Space Research Association GESTAR, Columbia, MD, USA \\ ${ }^{\mathrm{b}}$ NASA Goddard Space Flight Center, Code 613, Greenbelt, MD, USA \\ ${ }^{\mathrm{c}}$ Institute of Remote Sensing, University of Bremen, Bremen, Germany
}

\section{A R T I C L E I N F O}

\section{Article history:}

Received 22 March 2013

Received in revised form

10 June 2013

Accepted 11 June 2013

Available online 27 June 2013

Keywords:

Polarization

Modification of the discrete ordinates

method

Surface reflection

\begin{abstract}
A B S T R A C T
A new polarized radiative transfer code Atmospheric Polarization Computations (APC) is described. The code is based on separation of the diffuse light field into anisotropic and smooth (regular) parts. The anisotropic part is computed analytically. The smooth regular part is computed numerically using the discrete ordinates method. Vertical stratification of the atmosphere, common types of bidirectional surface reflection and scattering by spherical particles or spheroids are included. A particular consideration is given to computation of the bidirectional polarization distribution function (BPDF) of the waved ocean surface.
\end{abstract}

(c) 2013 Elsevier Ltd. All rights reserved.

\section{Introduction}

Polarization must be taken into account for any exact treatment of the problems of light scattering [9, p. 24]. The effect of polarization is important both for accurate computation of the intensity, I, (e.g., GOSAT project, http:// www.gosat.nies.go.jp/eng/gosat/page2.htm) and for retrieving optical properties of the scattering media from the measurements of polarization components of the Stokes vector $\overrightarrow{\mathbf{S}}=\left[\begin{array}{llll}I & Q & U & V\end{array}\right]$ (POLDER-2 instrument onboard PARASOL microsatellite http://smsc.cnes.fr/PARASOL).

Different methods are used for numerical simulations of polarized light. Chandrasekhar [9] proposed the solution for pure Rayleigh scattering. An adding-doubling method was used in the codes developed by Hovenier [20], Hansen and Travis [18], and Evans and Stephens [13]. The method of spherical harmonics was generalized for the case of polarization by Benassi et al. [7], Garcia and Siewert [14], Ustinov [59], yet this powerful method is not widely used today for polarization computations. Other solution techniques include the invariant embedding [40],

\footnotetext{
* Corresponding author at: NASA Goddard Space Flight Center, Code 613, Greenbelt, MD, USA. Tel.: +1 301614 5153; fax: +1 3016146307.

E-mail address: sergey.v.korkin@nasa.gov (S.V. Korkin).
}

the Monte-Carlo [26,37,42], and successive orders of scattering [34] methods. The low order of scattering approximations [20,46] are useful for the cases with optically thin or strongly absorbing medium. The above list of methods is not complete. The reader is referred to the monograph by Hovenier et al. [22, paragraph 5.1] for more detail. As this monograph is frequently referenced in this paper, we will further use an acronym "TPL" ("Transfer of polarized light...") for convenience. We will also use an acronym "ART" ("Analysis of the radiative transfer...") for another paper [30] frequently referenced herein. In general, the advantages and disadvantages of different methods are similar for the scalar and vector cases as discussed by Lenoble [33].

In this paper, the discrete ordinates method (DOM) is used for solution of the transfer problem. DOM was developed by Chandrasekhar [9] and references therein. It has been used in different vector codes such as SCIATRAN [52,53], PStar [47], VLIDORT [56] and others. DOM naturally includes the surface reflection in the form of Mark [38]. The theoretical basis of DOM as applied to the vector radiative transfer equation (VRTE) was developed by Siewert [55] and Garcia and Siewert [15].

DOM is based on numerical evaluation of the scattering integral of VRTE using the Gaussian quadrature. Double Gauss rule is often used [57]. Given the number of Gaussian 
nodes (so called "streams") $2 N$, the system of $8 N$ linear differential equations are to be solved ( $4 \mathrm{~N}$ equations per hemisphere). By analogy with the scalar case, the VRTE is expanded into a Fourier series over azimuthal angle. For each of $m=0 \ldots M$ azimuthal harmonics, the system of $8 \mathrm{~N}$ equations is solved independently. The expansion into Fourier series is essentially related to the expansion of the phase matrix into Legendre series. In DOM, the number of Legendre expansion terms $K$ and the number of nodes $2 \mathrm{~N}$ are not independent. The rule $2 N=K$ gives the best result according to the properties of the Gauss scheme [58]. The number $K$ can be very large in case of scattering by large particles such as ice crystals, coarse fraction of aerosol or hydrosol. This translates into a large number of linear equations in the system which increases computational time and makes problem ill-conditioned. In the vector case, the number of equations is four times as large as in the scalar case and the above mentioned problems become more severe.

The computational time and ill-conditionality can be reduced by truncating the number of the Fourier terms $(M)$ and the order of Gauss scheme $(N)$. Physically it means
$[4,5,28]$. The reader is referred to [28] for comparative analysis of the MVDOM code and other codes in case of coarse aerosol and clouds. APC is a further development of MVDOM suitable for simulations and applied analysis: it includes vertical stratification of the atmosphere, built-in spherical/spheroidal aerosol models [12], and common land bidirectional reflectance models or a model of windruffled ocean surface as the bottom boundary. More details on the new features of APC are provided below.

The paper is structured as follows: Section 2 defines the problem. The anisotropic and regular parts are considered in Sections 3 and 4 respectively. The algorithm of computation of the surface reflection is described in detail in Section 5. Several computational examples are given in Section 6 . The paper is concluded with the summary.

\section{Formulation of the problem}

The boundary-value problem for the polarized monochromatic radiation scattered in the plane parallel atmosphere with no internal sources of energy is given by

$$
\left\{\begin{array}{l}
\mu \frac{\vec{\partial}_{(\tau, \mu, \varphi)}}{\partial \tau}+\overrightarrow{\mathbf{S}}(\tau, \mu, \varphi)=\frac{\omega_{0}}{4 \pi} \int_{0}^{2 \pi} \int_{-1}^{1} \mathbf{P}\left(\tau, \mu, \varphi, \mu^{\prime}, \varphi^{\prime}\right) \overrightarrow{\mathbf{S}}\left(\tau, \mu^{\prime}, \varphi^{\prime}\right) d \mu^{\prime} d \varphi^{\prime}+\overrightarrow{\mathbf{Q}}\left(\tau, \mu, \varphi, \mu_{0}, \varphi_{0}\right), \\
\overrightarrow{\mathbf{S}}\left(0, \mu_{+}, \varphi\right)=\overrightarrow{\mathbf{0}} ; \quad \overrightarrow{\mathbf{S}}\left(\tau_{0}, \mu_{-}, \varphi\right)=\frac{1}{\pi} \int_{0}^{2 \pi} \int_{0}^{1} \mathbf{R}\left(\mu, \varphi, \mu^{\prime}, \varphi^{\prime}\right) \overrightarrow{\mathbf{S}}\left(\tau_{0}, \mu^{\prime}, \varphi^{\prime}\right) \mu^{\prime} d \mu^{\prime} d \varphi^{\prime}+\overrightarrow{\mathbf{D}}\left(\tau_{0}, \mu_{0}, \varphi_{0}\right)
\end{array}\right.
$$

that the radiation field must be a smooth function of the view zenith (VZA) and azimuthal angles. In order to achieve this goal, the Delta- $M$ method [60] combined with the single scattering correction [45] is used. While this correction significantly reduces the number of streams $N$ and number of harmonics $M$, it also changes the phase function causing substantial error in the aureole area $[45,31]$. Recent investigations revealed an error up to $4.5 \%$ [10] caused by truncation of the phase matrix. Thus, any vector computations with modified phase matrix should be done with caution.

This paper uses a different approach. Rather than changing the phase matrix, it splits the light field into the anisotropic and smooth parts. The Stokes vector of the diffuse light field is presented in the following form:

$\overrightarrow{\mathbf{S}}(\tau, \mu, \varphi)=\overrightarrow{\mathbf{S}}_{A}(\tau, \mu, \varphi)+\overrightarrow{\mathbf{S}}_{R}(\tau, \mu, \varphi)$,

where $\overrightarrow{\mathbf{S}}_{A}$ and $\overrightarrow{\mathbf{S}}_{R}$ are the anisotropic [2] and the regular parts [4], as defined below, $\tau$ is the optical depth (OD), $\mu$ is the cosine of the VZA, $\varphi$ is the relative azimuth. VZA, $\theta$, is defined following TPL, Eq. (3.18): $\mu=-\cos \theta$. Eq. (1) avoids truncation of the phase function. Korkin et al. [30,31] have recently investigated approach Eq. (1) for the scalar case and showed that it yields a high accuracy solution at low orders $N$ and $M$, similarly to the TMS method [45], but with accurate representation of the aureole.

The approach Eq. (1) is the basis of the new Atmospheric Polarization Computations (APC) code. In scalar case, this approach has been investigated by Romanova [51], Irvine [24], and Budak et al. [6], and references therein. In vector form, Eq. (1) was used in code MVDOM
In Eq. (2), a vector with positive cosine $\mu$ is pointed down to the surface, $\mu_{0}$ is the cosine of solar zenith angle (SZA), $\omega_{0}$ is the single scattering albedo, $\tau_{0}$ is the total OD of the atmosphere, $0 \leq \tau \leq \tau_{0}, \mathbf{P}\left(\tau, \mu, \varphi, \mu^{\prime}, \varphi^{\prime}\right)$ is the phase matrix, $\mathbf{R}\left(\mu, \varphi, \mu^{\prime}, \varphi^{\prime}\right)$ is the bidirectional polarized reflectance distribution function (BPDF) that accounts for effects of polarization. The vector of zeros is noted as $\overrightarrow{\mathbf{0}}$. Further in the text, the bold symbols indicate square matrices, bold symbols with arrows indicate vectors. $\overrightarrow{\mathbf{Q}}\left(\tau, \mu, \varphi, \mu_{0}, \varphi_{0}\right)$ is the free term of the source function

$\overrightarrow{\mathbf{Q}}\left(\tau, \mu, \varphi, \mu_{0}, \varphi_{0}\right)=\frac{\omega_{0}}{4 \pi} \mathbf{P}\left(\tau, \mu, \varphi, \mu_{0}, \varphi_{0}\right) \overrightarrow{\mathbf{S}}_{0} \exp \left(-\tau / \mu_{0}\right)$,

which represents the direct light beam. For brevity, the free term $\overrightarrow{\mathbf{Q}}\left(\tau, \mu, \varphi, \mu_{0}, \varphi_{0}\right)$ in Eq. (2) (or $\overrightarrow{\mathbf{J}}$ in Eq. (30) below) is further called "the source function." The term $\overrightarrow{\mathbf{D}}\left(\tau_{0}, \mu_{0}, \varphi_{0}\right)$ describes reflection of the direct solar beam:

$\overrightarrow{\mathbf{D}}\left(\tau_{0}, \mu_{0}, \varphi_{0}\right)=1 / \pi \mathbf{R}\left(\mu, \varphi, \mu_{0}, \varphi_{0}\right) \mu_{0} \exp \left(-\tau_{0} / \mu_{0}\right) \overrightarrow{\mathbf{S}}_{0}$

Eq. (2) is written in the coordinate system defined by Hovenier and van der Mee [21, Eq. (170) and Figs. 2 and 3]. The solution of Eq. (2) is decomposed into azimuthal Fourier series following Siewert [55]. For the case of unpolarized collimated beam $\left(\overrightarrow{\mathbf{S}}_{0}=\left[\begin{array}{llll}1 & 0 & 0 & 0\end{array}\right]\right)$ incident on the top of atmosphere (TOA), the Fourier decomposition of the solution is

$\overrightarrow{\mathbf{S}}(\tau, \mu, \varphi)=\sum_{m=0}^{M}\left(2-\delta_{0, m}\right) \mathbf{\Phi}(m \varphi) \overrightarrow{\mathbf{S}}^{m}(\tau, \mu)$, 
where

$\boldsymbol{\Phi}(\varphi)=\operatorname{diag}\left[\begin{array}{llll}\cos \varphi & \cos \varphi & \sin \varphi & \sin \varphi\end{array}\right] ; \quad \delta_{0, m}=\left\{\begin{array}{lr}1, & m=0, \\ 0, & m \neq 0 .\end{array}\right.$

The $m$-th Fourier moment of the source function Eq. (3) is given by

$\overrightarrow{\mathbf{Q}}^{m}\left(\tau, \mu, \mu_{0}\right)=\frac{\omega_{0}}{2} \mathbf{P}^{m}\left(\tau, \mu, \mu_{0}\right) \overrightarrow{\mathbf{S}}_{0} \exp \left(-\tau / \mu_{0}\right)$

where the $m$-th moment of the phase matrix $\mathbf{P}\left(\tau, \mu, \mu^{\prime}, \varphi, \varphi^{\prime}\right)$ is

$\mathbf{P}^{m}\left(\tau, \mu, \mu^{\prime}\right)=\sum_{k=m}^{K}(2 k+1) \mathbf{P}_{k}^{m}(\mu) \mathbf{F}_{k}(\tau) \mathbf{P}_{k}^{m}\left(\mu^{\prime}\right)$.

Here, $\mathbf{P}_{k}^{m}(\mu)$ contains $Q_{k}^{m}(\mu), R_{k}^{m}(\mu)$ and $T_{k}^{m}(\mu)$ polynomials, related to the generalized spherical functions $P_{m, n}^{k}(\mu)[16,55]$. $K$ is the maximum considered order of the polynomial. Note, that all expansion terms of the phase function are used in $\overrightarrow{\mathbf{Q}}^{m}\left(\tau, \mu, \mu_{0}\right)$, Eq. (7), and $\overrightarrow{\mathbf{J}}^{m}\left(\tau, \mu_{i}, \mu_{0}\right)$, Eq. (31).

The scattering matrix and the matrix expansion moments (see Eq. (8)), respectively, are written as

$\mathbf{F}(\tau, \Theta)=\left[\begin{array}{cccc}a_{1} & b_{1} & 0 & 0 \\ b_{1} & a_{2} & 0 & 0 \\ 0 & 0 & a_{3} & b_{2} \\ 0 & 0 & -b_{2} & a_{4}\end{array}\right], \quad \mathbf{F}_{k}(\tau)=\left[\begin{array}{cccc}\alpha_{1}^{k} & \beta_{1}^{k} & 0 & 0 \\ \beta_{1}^{k} & \alpha_{2}^{k} & 0 & 0 \\ 0 & 0 & \alpha_{3}^{k} & \beta_{2}^{k} \\ 0 & 0 & -\beta_{2}^{k} & \alpha_{4}^{k}\end{array}\right]$,

where $\Theta$ is the scattering angle.

Van de Hulst [23, paragraph 5.22], case 6 has formulated the assumptions behind the symmetry properties of the scattering matrix, Eq. (9). The computational expressions for each element of $\mathbf{F}_{k}(\tau)$, Eq. (9), are known from $[54,41]$ and TPL, paragraph 2.8. For Rayleigh scattering we use Eqs. (3.122) and (3.132) from TPL.

The scattering matrix Eq. (9) is used for spherical particles and spheroids. The APC code uses the model of spheres and spheroids described in Dubovik et al. [12].

The phase matrix $\mathbf{P}\left(\tau, \mu, \varphi, \mu^{\prime}, \varphi^{\prime}\right)$ takes into account the effect of rotation of the reference plane of the Stokes vector. The positive angle of rotation is defined following TPL, p. 11. Namely, angle of rotation, $\sigma$, is considered positive if rotation is performed in the anti-clockwise direction when looking in the direction of propagation. Thus, the rotation matrix is (TPL, Eq. (1.51))

$\mathbf{L}(\sigma)=\left[\begin{array}{cccc}1 & 0 & 0 & 0 \\ 0 & \cos 2 \sigma & \sin 2 \sigma & 0 \\ 0 & -\sin 2 \sigma & \cos 2 \sigma & 0 \\ 0 & 0 & 0 & 1\end{array}\right], \quad \mathbf{L}(\pi-\sigma)=\mathbf{L}(-\sigma)$,

and the phase matrix is defined following TPL, Eqs. (3.7) and (3.22),

$\mathbf{P}\left(\tau, \mu, \varphi, \mu^{\prime}, \varphi^{\prime}\right)=\mathbf{L}\left(\pi-\sigma_{2}\right) \mathbf{F}(\tau, \Theta) \mathbf{L}\left(-\sigma_{1}\right)$.

In order to evaluate the scattering integral, either the scattering or the rotation matrix is to be diagonalized. The former is possible for the molecular scattering in the Chandrasekhar's basis [9, Eq. (162)]. In general case, the circular CP-basis was proposed by Kuščer and Ribarič [32]. The following notation is used here: $\mathbf{T}_{C}$ is the matrix of transformation from the Stokes basis (SP - Stokes polarization) to the circular one ( $\mathrm{CP}$ - circular polarization) and $\mathbf{T}_{S}=\mathbf{T}_{C}^{-1}$ gives the inverse transform. Following (Eqs. (1.78) and (1.80)) from TPL:

$\mathbf{T}_{C}=\frac{1}{2}\left[\begin{array}{cccc}0 & 1 & i & 0 \\ 1 & 0 & 0 & 1 \\ 1 & 0 & 0 & -1 \\ 0 & 1 & -i & 0\end{array}\right] ; \quad \mathbf{T}_{S}=\left[\begin{array}{cccc}0 & 1 & 1 & 0 \\ 1 & 0 & 0 & 1 \\ -i & 0 & 0 & i \\ 0 & 1 & -1 & 0\end{array}\right] ; \quad \begin{aligned} & \overrightarrow{\mathbf{S}}_{C P}=\mathbf{T}_{C} \overrightarrow{\mathbf{S}}_{S P}, \\ & \mathbf{M}_{C P}=\mathbf{T}_{C} \mathbf{M}_{S P} \mathbf{T}_{S},\end{aligned}$

where $\mathbf{M}_{C P}$ and $\mathbf{M}_{S P}$ are the representations of an arbitrary matrix in $\mathrm{CP}$ and SP basis, respectively.

The definition Eq. (12), used in this paper, is not unique. For example, Ustinov [59, Appendix A, Eq. (8)] proposed a different equivalent formulation.

This section provided the main definitions and the minimum theoretical background. The next two sections will describe solution of Eqs. (1) and (2) using the discrete ordinates method.

\section{The anisotropic part}

The anisotropic part in vector form has been derived and analyzed in detail in Astakhov et al. [2] based on properties of the expansion coefficients of $\overrightarrow{\mathbf{S}}_{A}(\tau, \mu, \varphi)$ over $P_{m, n}^{k}(\mu)$. This section highlights the necessary properties of the anisotropic part based on analogy with the scalar case. Only a natural (solar) incident beam is considered.

The anisotropic part, $\overrightarrow{\mathbf{S}}_{A}(\tau, \mu, \varphi)$, is computed in the coordinate system centered in the solar beam direction (see Fig. 1 and ART, Section 2. SAM: evaluation of anisotropic part). It is convenient to compute $\overrightarrow{\mathbf{S}}_{A}(\tau, \mu, \varphi)$ as a function of scattering angle $\Theta=\operatorname{acos}(\nu)$ where

$\nu=\cos (\Theta)=\mu \mu_{0}+\sqrt{1-\mu^{2}} \sqrt{1-\mu_{0}^{2}} \cos \varphi$,

i.e. $\overrightarrow{\mathbf{S}}_{A}(\tau, \mu, \varphi)=\overrightarrow{\mathbf{S}}_{A}(\tau, \nu)$. In the aureole area, $\nu \approx 1, \overrightarrow{\mathbf{S}}_{A}(\tau, \nu)$ is close to the accurate numerical solution and almost symmetric around the $\nu=1$ direction. Similar to the scalar case, it only needs the $m=0$ Fourier term. Although the $\overrightarrow{\mathbf{S}}_{A}(\tau, \nu)$ error can be large outside the aureole region, this error is a smooth function of angles compensated later in the regular part, $\overrightarrow{\mathbf{S}}_{R}(\tau, \mu, \varphi)$.

Following the scalar case [17,33 (Eq. (4.46))], [3], and ART, Eq. (3), the VRTE in the small angle approximation is

$$
\begin{aligned}
\mu_{0} & \frac{\partial \overrightarrow{\mathbf{S}}_{A}(\tau, \mu, \varphi)}{\partial \tau}+\overrightarrow{\mathbf{S}}_{A}(\tau, \mu, \varphi) \\
= & \frac{\omega_{0}}{4 \pi} \int_{0}^{2 \pi} \int_{-1}^{1} \mathbf{P}\left(\tau, \mu, \varphi, \mu^{\prime}, \varphi^{\prime}\right) \overrightarrow{\mathbf{S}}_{A}\left(\tau, \mu^{\prime}, \varphi^{\prime}\right) d \mu^{\prime} d \varphi^{\prime} \\
& +\overrightarrow{\mathbf{Q}}\left(\tau, \mu, \varphi, \mu_{0}, \varphi_{0}\right),
\end{aligned}
$$

where $\overrightarrow{\mathbf{Q}}\left(\tau, \mu, \varphi, \mu_{0}, \varphi_{0}\right)$ is defined in Eq. (3). Eq. (14) is subject to the boundary condition $\overrightarrow{\mathbf{S}}_{A}(0, \mu, \varphi)=\overrightarrow{\mathbf{0}}$ on the TOA only.

Using the orthogonal properties of the generalized spherical functions, the addition theorem (TPL, Appendix B), and 


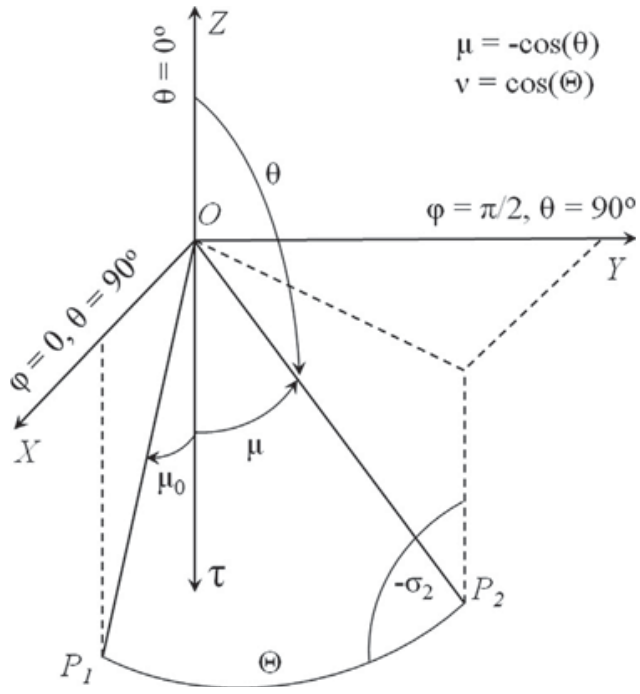

Fig. 1. The right-handed Cartesian coordinate system. $\mathrm{OP}_{1}$ is the solar beam direction. $\mathrm{OP}_{2}$ is the direction of observation (scattering). $\mathrm{ZOX}$ (the same as $\mathrm{ZOP}_{1}$ ) is the principal plane. $\mathrm{P}_{1} \mathrm{OP}_{2}$ is the scattering plane. $\mathrm{ZOP}_{2}$ is the plane of observation. Positive values of $\mu$ are measured from the positive $\tau$-axis (optical depth). The case considered in this figure is identical to Fig. 3.3 from TPL.

the expansion of the VRTE solution [59, Eq. (14)].

$\overrightarrow{\mathbf{S}}_{A}^{C P}(\tau, \nu)=\sum_{k=0}^{K_{\max }} \frac{2 k+1}{4 \pi} \mathbf{Y}_{k}^{m=0}(\nu) \overrightarrow{\mathbf{C}}_{k}(\tau)$

over the generalized spherical functions (in matrix form)

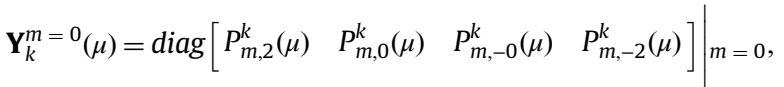

yields the system of equations for the expansion coefficients which is similar to that obtained by Ustinov [59, Eq. (18)]

$\mu_{0} d \overrightarrow{\mathbf{C}}_{k} / d \tau+\overrightarrow{\mathbf{C}}_{k}=\omega_{0} \mathbf{X}_{k} \overrightarrow{\mathbf{C}}_{k}+\omega_{0} \exp \left(-\tau / \mu_{0}\right) \mathbf{X}_{k} \overrightarrow{\mathbf{C}}_{0}$

Recall that Eq. (15) with $m=0$ is valid only in the coordinate system centered in the solar beam direction.

In Eq. (17), $\overrightarrow{\mathbf{C}}_{0}$ is defined by the boundary conditions on the TOA

$\overrightarrow{\mathbf{C}}_{0}=\mathbf{T}_{C} \overrightarrow{\mathbf{S}}_{0}=\left[\begin{array}{llll}0 & 1 / 2 & 1 / 2 & 0\end{array}\right]$

and

$\mathbf{X}_{k}=\mathbf{T}_{C} \mathbf{F}_{k} \mathbf{T}_{S}$.

The solution of the VRTE in the small angle approximation, Eq. (14), and in CP basis is

$\overrightarrow{\mathbf{C}}_{k}(\tau)=\left[\exp \left(-\left(\mathbf{1}-\omega_{0} \mathbf{X}_{k}\right) \tau / \mu_{0}\right)-\exp \left(-\tau / \mu_{0}\right) \mathbf{1}\right] \overrightarrow{\mathbf{C}}_{0}(\tau)$,

where $\mathbf{1}$ is the unit matrix. Eq. (20) is transferred to the SP-basis using Eq. (12)

$$
\begin{aligned}
\overrightarrow{\mathbf{S}}_{A}(\tau, \nu) & =\overrightarrow{\mathbf{S}}_{A}^{S P}(\tau, \nu)=\mathbf{T}_{S} \overrightarrow{\mathbf{S}}_{A}^{C P}(\tau, \nu) \\
& =\mathbf{T}_{S} \sum_{k=0}^{K} \frac{2 k+1}{4 \pi} \mathbf{Y}_{k}(\nu) \mathbf{T}_{C} \mathbf{T}_{S} \overrightarrow{\mathbf{C}}_{k}(\tau) \mathbf{T}_{C} \mathbf{T}_{S} \overrightarrow{\mathbf{C}}_{0}
\end{aligned}
$$

The next equation gives a useful relationship for the matrix exponential that follows from $\mathbf{T}_{S} \mathbf{T}_{C}=\mathbf{1}$

$\mathbf{T}_{S} \exp \left(\mathbf{X}_{k}\right) \mathbf{T}_{C}=\exp \left(\mathbf{T}_{S} \mathbf{X}_{k} \mathbf{T}_{C}\right)=\exp \left(\mathbf{F}_{k}\right)$

The matrix polynomial is transformed as follows:

$\mathbf{T}_{S} \mathbf{Y}_{k}^{0}(\nu) \mathbf{T}_{C}=\mathbf{P}_{k}^{0}(\nu)=\operatorname{diag}\left[Q_{k}^{0}(\nu) \quad R_{k}^{0}(\nu) \quad R_{k}^{0}(\nu) \quad Q_{k}^{0}(\nu)\right]$.

Eqs. (22) and (23) give the expression for the anisotropic part in the SP basis and in the coordinate system centered in the solar beam direction

$\overrightarrow{\mathbf{S}}_{A}(\tau, \nu)=\sum_{k=0}^{K_{\max }} \frac{2 k+1}{4 \pi} \mathbf{P}_{k}^{0}(\nu) \mathbf{Z}_{k}(\tau) \overrightarrow{\mathbf{S}}_{0}$,

where

$\mathbf{Z}_{k}(\tau)=\left[\exp \left(-\left(\mathbf{1}-\omega_{0} \mathbf{F}_{k}\right) \tau / \mu_{0}\right)-\exp \left(-\tau / \mu_{0}\right) \mathbf{1}\right]$.

Eq. (25) is computed using the singular value decomposition (SVD). Under the natural illumination, Eq. (24) contains only two parameters

$\overrightarrow{\mathbf{S}}_{A}(\tau, \nu)=\left[\begin{array}{llll}I_{A}(\tau, \nu) & Q_{A}(\tau, \nu) & 0 & 0\end{array}\right]$.

It follows from Eq. (24) that $\vec{S}_{A}(\tau, \nu)$ is defined with respect to the plane of scattering $\mathrm{P}_{1} \mathrm{OP}_{2}$ (see Fig. 1). The VRTE Eq. (2) is solved with respect to the plane formed by the vector of normal, and the view direction $\left(\mathrm{ZOP}_{2}\right.$ in Fig. 1). In order to match these two coordinate systems, rotator Eq. (10) is used exactly as it is used under the scattering integral. Following the analogy with the Eq. (3.7) from TPL

$$
\begin{gathered}
\overrightarrow{\mathbf{S}}_{A}(\tau, \nu)=\mathbf{R}\left(\pi-\sigma_{2}\right)\left[\sum_{k=0}^{K_{\max }} \frac{2 k+1}{4 \pi} \mathbf{P}_{k}(\nu) \mathbf{Z}_{k}(\tau)\right] \mathbf{R}\left(-\sigma_{1}\right) \overrightarrow{\mathbf{S}}_{0} \\
\quad=\mathbf{R}\left(-\sigma_{2}\right) \overrightarrow{\mathbf{S}}_{A}(\tau, \nu)=\left[\begin{array}{llll}
I_{A}(\tau, \nu) & Q_{A}(\tau, \nu) & U_{A}(\tau, \nu) & 0
\end{array}\right]
\end{gathered}
$$

The angle of rotation, $\sigma_{2}$ (Fig. 1 ), is computed using the sines and the cosines rule for angles [61]. Eqs. (3.11), (3.12), (3.14), and (3.23) from TPL provide the complete set of equations for computation of $\sigma_{2}$. Analytically it is convenient to transform Eq. (15) to the normal coordinate system in the $\mathrm{CP}$ basis where the rotation matrix has the diagonal form, and then to perform the CP-to-SP transformation. In the final form, the anisotropic part is expressed as follows [5, Eq. (15)]:

$$
\begin{aligned}
& \overrightarrow{\mathbf{S}}_{A}\left(\tau, \mu, \mu_{0}\right)=\sum_{m=0}^{k}\left(2-\delta_{0, m}\right) \overrightarrow{\mathbf{S}}_{A}^{m}\left(\tau, \mu, \mu_{0}\right) \\
& =\sum_{m=0}^{k}\left(2-\delta_{0, m}\right) \sum_{k=0}^{K_{\max }} \frac{2 k+1}{4 \pi} \boldsymbol{\Phi}(m \varphi) \mathbf{P}_{k}^{m}(\mu) \mathbf{Z}_{k}(\tau) \mathbf{P}_{k}^{m}\left(\mu_{0}\right) \overrightarrow{\mathbf{S}}_{0},
\end{aligned}
$$

where $\boldsymbol{\Phi}(m \varphi)$ and $\mathbf{Z}_{k}(\tau)$ are defined by Eq. (6) and (25) respectively.

The form of both Eqs. (24) and (28) is valid for the vertically stratified case, however $\mathbf{Z}_{k}(\tau)$ needs to be changed. Assuming the case of two layers with $\tau^{(1)}$ as the optical thickness of the top layer and the optical parameters of two layers defined as $\omega_{0}^{(1)}, \mathbf{F}_{k}^{(1)}$ and $\omega_{0}^{(2)}, \mathbf{F}_{k}^{(2)}$ 
respectively, one can write

$\mathbf{Z}_{k}\left(\tau \geq \tau_{1}\right)=\exp \left(-\left(\mathbf{1}-\omega_{0}^{(2)} \mathbf{F}_{k}^{(2)}\right)\left(\tau-\tau_{1}\right) / \mu_{0}\right) \exp \left(-\left(\mathbf{1}-\omega_{0}^{(1)} \mathbf{F}_{k}^{(1)}\right) \tau_{1} / \mu_{0}\right)-\exp \left(-\tau / \mu_{0}\right) \mathbf{1}$

similar to the scalar case [6, Eq. (5.77)].

This section provided formulation for the anisotropic part using two different coordinate systems and the smallangle solution within the forward hemisphere in the coordinate system of the incident beam. The anisotropic part in the standard coordinate system (with respect to the normal) is used next to formulate the bottom boundary condition and the source term for the regular part. The difference between the total VRTE solution and the described anisotropic part is considered in the next section.

\section{Regular part}

This section describes the equation and the method of numerical solution for the regular part, $\overrightarrow{\mathbf{S}}_{R}(\tau, \mu, \varphi)$. Eq. (1) is substituted in Eq. (2), and expansion Eq. (5) is used for the regular part. There is no particular difference with the scalar case at this step (ART, Eq. (9)) except for evaluation of the scattering integral in $\mathrm{CP}$ basis following Siewert [55]. The system of $8 \mathrm{~N}$ ordinary differential equations $(i=1 \ldots$ $2 N$, four equations per each value of $i$ ) for the regular part is similar to the scalar case (ART, Eq. (16))

$$
\begin{aligned}
\mu_{i} & \frac{d \overrightarrow{\mathbf{S}}_{R}^{m}\left(\tau, \mu_{i}\right)}{d \tau}+\overrightarrow{\mathbf{S}}_{R}^{m}\left(\tau, \mu_{i}\right) \\
& =\frac{\omega_{0}}{2} \sum_{j=1}^{2 N} \sum_{k=m}^{K}(2 k+1) w_{j} \mathbf{P}_{k}^{m}\left(\mu_{i}\right) \mathbf{F}_{k}(\tau) \mathbf{P}_{k}^{m}\left(\mu_{j}\right) \overrightarrow{\mathbf{S}}_{R}^{m}\left(\tau, \mu_{j}\right)+\overrightarrow{\mathbf{J}}^{m}\left(\tau, \mu_{i}, \mu_{0}\right),
\end{aligned}
$$

where $w_{j}, \mu_{j}$ are the weights and nodes of the Double Gauss scheme respectively. Given the number of ordinates per sphere $2 \mathrm{~N}$, the number of phase matrix expansion terms under the scattering integral is $K=2 N<K_{\max }$ (see Eqs. (15) and (28)).

Using Eqs. (2) and (14), it is easy to write

$$
\begin{aligned}
\overrightarrow{\mathbf{J}}^{m}\left(\tau, \mu_{i}, \mu_{0}\right) & =\left(\mu_{0}-\mu_{i}\right) \frac{d \overrightarrow{\mathbf{S}}_{A}^{m}\left(\tau, \mu_{i}\right)}{d \tau} \\
& =\frac{\mu_{i}-\mu_{0}}{\mu_{0}} \sum_{k=0}^{K}(2 k+1) \mathbf{P}_{k}^{m}\left(\mu_{i}\right) \mathbf{Z}_{k}(\tau) \mathbf{P}_{k}^{m}\left(\mu_{0}\right) \overrightarrow{\mathbf{S}}_{0}
\end{aligned}
$$

where $\overrightarrow{\mathbf{S}}_{A}^{m}(\tau, \mu, \varphi)$ is given by Eq. (28). Similarly to (ART, Eq. (17)), the recurrence relation for $\mu \mathbf{P}_{k}^{m}(\mu)$ is not used here. This simplifies the expression for the source function Eq. (31) if compared with [6, Eq. (5.123)]. Eq. (30) is independent for each $m=0 \ldots M$ (Eq. (5)). For brevity, the upper (Fourier) index $m$ is omitted further in the text as well as the index " $R$ " that indicates the regular part. Also, $\mu^{-}$and $\mu^{+}$are used to indicate the directions with negative (upward) and positive (downward to the ground) cosines of the VZA. The boundary condition on the TOA is $\overrightarrow{\mathbf{S}}\left(\tau, \mu^{+}\right)=\overrightarrow{\mathbf{0}}$. The boundary condition at the surface, $\overrightarrow{\mathbf{S}}\left(\tau_{0}, \mu^{-}\right)$, is discussed in Section 5.

Eq. (30) is solved using SVD and the scaling transformation following Karp et al. [25] to improve conditioning of the system. For this purpose, Eq. (30) is rewritten in the matrix form:

$\frac{d \overrightarrow{\mathbf{S}}(\tau)}{d \tau}=-\mathbf{M}^{-1}(\mathbf{1}-\mathbf{X W}) \overrightarrow{\mathbf{S}}(\tau)+\overrightarrow{\mathbf{J}}\left(\tau, \mu_{0}\right)$,

where

$$
\begin{aligned}
& \overrightarrow{\mathbf{S}}(\tau)=\left[\overrightarrow{\mathbf{S}}\left(\tau, \mu_{i}\right)\right]_{i=1: 2 N}, \quad \overrightarrow{\mathbf{J}}\left(\tau, \mu_{0}\right)=\left[\overrightarrow{\mathbf{J}}^{m}\left(\tau, \mu_{i}, \mu_{0}\right) / \mu_{i}\right]_{i=1: 2 N}, \\
& \boldsymbol{\mu}_{i}=\operatorname{diag}\left[\begin{array}{llll}
\mu_{i} & \mu_{i} & \mu_{i} & \mu_{i}
\end{array}\right], \mathbf{M}=\operatorname{diag}\left[\begin{array}{lll}
\boldsymbol{\mu}_{1} & \cdots & \boldsymbol{\mu}_{2 N}
\end{array}\right], \\
& \mathbf{W}=\operatorname{diag}\left[\begin{array}{lll}
\mathbf{w}_{1} & \cdots & \mathbf{w}_{2 N}
\end{array}\right], \quad \mathbf{w}_{i}=\operatorname{diag}\left[\begin{array}{llll}
w_{i} & w_{i} & w_{i} & w_{i}
\end{array}\right], \\
& \mathbf{X}=\left[\frac{\omega_{0}}{2} \sum_{k=m}^{K}(2 k+1) \mathbf{P}_{k}^{m}\left(\mu_{i}\right) \mathbf{F}_{k}(\tau) \mathbf{P}_{k}^{m}\left(\mu_{j}\right)\right]_{i, j=1: 2 N} .
\end{aligned}
$$

Using the notation

$$
\mathbf{B}=\mathbf{M}^{-1}(\mathbf{1}-\mathbf{X W})
$$

and assuming the homogeneous layer, Eq. (32) is solved as [25, Eq. (8)]

$-\overrightarrow{\mathbf{S}}(0)+\exp \left(\mathbf{B} \tau_{0}\right) \overrightarrow{\mathbf{S}}\left(\tau_{0}\right)=\int_{0}^{\tau_{0}} \exp (\mathbf{B} \tau) \overrightarrow{\mathbf{J}}(\tau) d \tau$

Eqs. (20)-(32) from ART give the step-by-step transformation of Eq. (32) to the system in the matrix-operator form [49]:

$$
\left[\begin{array}{c}
\overrightarrow{\mathbf{S}}_{-}(0) \\
\overrightarrow{\mathbf{S}}_{+}\left(\tau_{0}\right)
\end{array}\right]=\left[\begin{array}{c}
\overrightarrow{\mathbf{J}}_{-} \\
\overrightarrow{\mathbf{J}}_{+}
\end{array}\right]+\left[\begin{array}{ll}
\mathbf{R}_{-} & \mathbf{T}_{-} \\
\mathbf{T}_{+} & \mathbf{R}_{+}
\end{array}\right]\left[\begin{array}{c}
\overrightarrow{\mathbf{S}}_{+}(0) \\
\overrightarrow{\mathbf{S}}_{-}\left(\tau_{0}\right)
\end{array}\right],
$$

where $\overrightarrow{\mathbf{S}}_{-}(0)$ and $\overrightarrow{\mathbf{S}}_{+}\left(\tau_{0}\right)$ are the solution for the radiation reflected from and transmitted through the layer, $\mathbf{R}_{ \pm}$and $\mathbf{T}_{+}$are the elements of the matrix that propagate the boundary conditions $\overrightarrow{\mathbf{S}}_{+}(0)=\overrightarrow{\mathbf{0}}$ and $\overrightarrow{\mathbf{S}}_{-}\left(\tau_{0}\right)$ through the layer. Eqs. (33)-(37) from ART give the step-by-step scaling transformation of the source vector $\left[\overrightarrow{\mathbf{J}}_{-} \overrightarrow{\mathbf{J}}_{+}\right]$. Only two features make the processing of the vector case different from the scalar one. First of all, the SVD gives complex values. They are computed by DGEEV subroutine from the LAPACK package. The second feature is that SVD should be used not only for Eq. (33), but for $\mathbf{Z}_{k}(\tau)$ in $\mathbf{J}(\tau)$, Eq. (34), as well. After that, the integral over $\tau$ is evaluated analytically. The SVD for $\mathbf{Z}_{k}(\tau)$ is performed separately for each 4-by-4 matrix exponential with a fixed $k$ in the preprocessing.

In general case, the atmosphere is divided into a number of homogeneous layers. The integration in Eq. (34) is performed for each layer [25, Eq. (8)]. Using two layers, (1) and (2), as an example, one can write

$$
\left\{\begin{array}{l}
{\left[\begin{array}{l}
\overrightarrow{\mathbf{S}}_{-}^{(1)}(0) \\
\overrightarrow{\mathbf{S}}_{+}^{(1)}\left(\tau_{e}\right)
\end{array}\right]=\left[\begin{array}{l}
\overrightarrow{\mathbf{J}}_{-}^{(1)} \\
\overrightarrow{\mathbf{J}}_{+}^{(1)}
\end{array}\right]+\left[\begin{array}{ll}
\mathbf{R}_{-}^{(1)} & \mathbf{T}_{-}^{(1)} \\
\mathbf{T}_{+}^{(1)} & \mathbf{R}_{+}^{(1)}
\end{array}\right]\left[\begin{array}{c}
\overrightarrow{\mathbf{S}}_{+}^{(1)}(0) \\
\overrightarrow{\mathbf{S}}_{-}^{(1)}\left(\tau_{e}\right)
\end{array}\right] ;} \\
{\left[\begin{array}{l}
\overrightarrow{\mathbf{S}}_{-}^{(2)}\left(\tau_{e}\right) \\
\overrightarrow{\mathbf{S}}_{+}^{(2)}\left(\tau_{0}\right)
\end{array}\right]=\left[\begin{array}{l}
\vec{J}_{-}^{(2)} \\
\overrightarrow{\mathbf{J}}_{+}^{(2)}
\end{array}\right]+\left[\begin{array}{ll}
\mathbf{R}_{-}^{(2)} & \mathbf{T}_{-}^{(2)} \\
\mathbf{T}_{+}^{(2)} & \mathbf{R}_{+}^{(2)}
\end{array}\right]\left[\begin{array}{l}
\overrightarrow{\mathbf{S}}_{+}^{(2)}\left(\tau_{e}\right) \\
\overrightarrow{\mathbf{S}}_{-}^{(2)}\left(\tau_{0}\right)
\end{array}\right] .}
\end{array}\right.
$$

The OD of the upper layer is assumed to be $\tau_{e}$, and the OD of the second (embedded) layer is assumed to be $\tau_{0}-\tau_{e}$. Using the continuity condition on the common boundary of two layers, one can write

$\overrightarrow{\mathbf{S}}_{+}^{(1)}\left(\tau_{e}\right)=\overrightarrow{\mathbf{S}}_{+}^{(2)}\left(\tau_{e}\right), \quad \overrightarrow{\mathbf{S}}_{-}^{(2)}\left(\tau_{e}\right)=\overrightarrow{\mathbf{S}}_{-}^{(1)}\left(\tau_{e}\right)$ 
Eq. (36) is solved with respect to $\overrightarrow{\mathbf{S}}_{-}^{(1)}(0)$ and $\overrightarrow{\mathbf{S}}_{+}^{(2)}\left(\tau_{0}\right)$ in Eq. (35). After that, the system Eq. (36) can be solved with respect to the field inside the media. Currently, the APC code computes the reflected and transmitted radiation at the interfaces of layers. The height profiles of air pressure and temperature are based on MODTRAN [27] and borrowed from code SHARM [36].

The APC code employs the idea of "natural" interpolation at an arbitrary viewing direction by including the required view angles as dummy nodes, $\mu_{d}$, into DOM scheme with zero weighting coefficients $w_{d}=0$ [8].

This section briefly described the method of numerical computation of the regular part. In general, it follows the scalar approach with the exception of complex SVD in the vector case. Also, two SVDs are necessary on both sides of Eq. (34) in order to perform the integration over OD analytically. The use of dummy nodes provides solution for the arbitrary directions. The surface boundary condition is formulated in the next section.

\section{Surface reflection}

The bottom boundary condition for the regular part is derived from Eqs. (1) and (2)

$$
\begin{aligned}
& \overrightarrow{\mathbf{S}}_{R}\left(\tau_{0}, \mu_{-}, \varphi\right)=-\overrightarrow{\mathbf{S}}_{A}\left(\tau_{0}, \mu_{-}, \varphi\right) \\
& \quad+\frac{1}{\pi} \int_{0}^{2 \pi} \int_{0}^{1} \mathbf{R}\left(\mu_{-}, \varphi, \mu^{\prime}, \varphi^{\prime}\right)\left[\overrightarrow{\mathbf{S}}_{A}\left(\tau_{0}, \mu^{\prime}, \varphi^{\prime}\right)\right. \\
& \left.\quad+\overrightarrow{\mathbf{S}}_{R}\left(\tau_{0}, \mu^{\prime}, \varphi^{\prime}\right)\right] \mu^{\prime} d \mu^{\prime} d \varphi^{\prime} \\
& \quad+\frac{1}{\pi} \mathbf{R}\left(\mu_{-}, \varphi, \mu_{0}, \varphi_{0}\right) \mu_{0} \exp \left(-\tau_{0} / \mu_{0}\right) \overrightarrow{\mathbf{S}}_{0}
\end{aligned}
$$

The matrix-operator technique, Eq. (35), uses the azimuthal (Fourier) moments, $\overrightarrow{\mathbf{S}}_{-}\left(\tau_{0}\right)$, of Eq. (38)

$$
\begin{aligned}
& \overrightarrow{\mathbf{S}}_{R}^{m}\left(\tau_{0}, \mu_{-}\right)=-\overrightarrow{\mathbf{S}}_{A}^{m}\left(\tau_{0}, \mu_{-}\right) \\
& \quad+\frac{1}{\pi} \int_{0}^{1} \mathbf{R}^{m}\left(\mu_{-}, \mu^{\prime}\right)\left[\overrightarrow{\mathbf{S}}_{A}^{m}\left(\tau_{0}, \mu^{\prime}\right)+\overrightarrow{\mathbf{S}}_{R}^{m}\left(\tau_{0}, \mu^{\prime}\right)\right] \mu^{\prime} d \mu^{\prime} \\
& \quad+\mathbf{R}^{m}\left(\mu_{-}, \mu_{0}\right) \mu_{0} \exp \left(-\tau_{0} / \mu_{0}\right) \overrightarrow{\mathbf{S}}_{0} / \pi .
\end{aligned}
$$

The integration in Eq. (39) is performed numerically. In the framework of DOM, Eq. (39) is rewritten as

$$
\overrightarrow{\mathbf{S}}_{-}\left(\tau_{0}\right)=\overrightarrow{\mathbf{S}}_{R}^{-}\left(\tau_{0}\right)=-\overrightarrow{\mathbf{S}}_{A}^{-}\left(\tau_{0}\right)+\mathbf{R}_{B}\left(\overrightarrow{\mathbf{S}}_{A}^{+}\left(\tau_{0}\right)+\overrightarrow{\mathbf{S}}_{R}^{+}\left(\tau_{0}\right)\right)+\overrightarrow{\mathbf{D}}_{B},
$$

where

$$
\begin{aligned}
& \overrightarrow{\mathbf{S}}_{R}^{ \pm}\left(\tau_{0}\right)=\left[\begin{array}{l}
\overrightarrow{\mathbf{S}}_{R}^{m}\left(\tau_{0}, \mu_{1}^{ \pm}\right) \\
\overrightarrow{\mathbf{S}}_{R}^{m}\left(\tau_{0}, \mu_{2}^{ \pm}\right) \\
\cdots \\
\overrightarrow{\mathbf{S}}_{R}^{m}\left(\tau_{0}, \mu_{N}^{ \pm}\right)
\end{array}\right] ; \quad \overrightarrow{\mathbf{S}}_{A}^{ \pm}\left(\tau_{0}\right)=\left[\begin{array}{l}
\overrightarrow{\mathbf{S}}_{A}^{m}\left(\tau_{0}, \mu_{1}^{ \pm}\right) \\
\overrightarrow{\mathbf{S}}_{A}^{m}\left(\tau_{0}, \mu_{2}^{ \pm}\right) \\
\cdots \\
\overrightarrow{\mathbf{S}}_{A}^{m}\left(\tau_{0}, \mu_{N}^{ \pm}\right)
\end{array}\right] ; \\
& \overrightarrow{\mathbf{D}}_{B}=\frac{\mu_{0}}{\pi} \exp \left(-\frac{\tau_{0}}{\mu_{0}}\right)\left[\begin{array}{l}
\mathbf{R}^{m}\left(\mu_{1}^{-}, \mu_{0}\right) \overrightarrow{\mathbf{S}}_{0} \\
\mathbf{R}^{m}\left(\mu_{2}^{-}, \mu_{0}\right) \overrightarrow{\mathbf{S}}_{0} \\
\cdots \\
\mathbf{R}^{m}\left(\mu_{N}^{-}, \mu_{0}\right) \overrightarrow{\mathbf{S}}_{0}
\end{array}\right] ;
\end{aligned}
$$

$\mathbf{R}_{B}=\frac{1}{\pi}\left[\begin{array}{llll}\mathbf{R}^{m}\left(\mu_{1}^{-}, \mu_{1}^{+}\right) \mu_{1}^{+} w_{1} & \mathbf{R}^{m}\left(\mu_{1}^{-}, \mu_{2}^{+}\right) \mu_{2}^{+} w_{2} & \cdots & \mathbf{R}^{m}\left(\mu_{1}^{-}, \mu_{N}^{+}\right) \mu_{N}^{+} w_{N} \\ \mathbf{R}^{m}\left(\mu_{2}^{-}, \mu_{1}^{+}\right) \mu_{1}^{+} w_{1} & \mathbf{R}^{m}\left(\mu_{2}^{-}, \mu_{2}^{+}\right) \mu_{2}^{+} w_{2} & \cdots & \mathbf{R}^{m}\left(\mu_{2}^{-}, \mu_{N}^{+}\right) \mu_{N}^{+} w_{N} \\ \cdots & \cdots & \cdots & \cdots \\ \mathbf{R}^{m}\left(\mu_{N}^{-}, \mu_{1}^{+}\right) \mu_{1}^{+} w_{1} & \mathbf{R}^{m}\left(\mu_{N}^{-}, \mu_{2}^{+}\right) \mu_{2}^{+} w_{2} & \cdots & \mathbf{R}^{m}\left(\mu_{N}^{-}, \mu_{N}^{+}\right) \mu_{N}^{+} w_{N}\end{array}\right]$

Eq. (40) is substituted in Eq. (35) that now contains only two unknowns: $\overrightarrow{\mathbf{S}}_{+}\left(\tau_{0}\right)$ and $\overrightarrow{\mathbf{S}}_{-}(0)$. The last equation in Eq. (35) is solved for $\overrightarrow{\mathbf{S}}_{+}\left(\tau_{0}\right)$ analytically.

In order to perform computations, the Mueller matrix of the surface $\mathbf{R}\left(\mu, \varphi, \mu^{\prime}, \varphi^{\prime}\right)$ is needed. The following Mueller matrix is used to include scalar case in vector computations

$\mathbf{R}\left(\mu, \varphi, \mu^{\prime}, \varphi^{\prime}\right)=\operatorname{diag}\left[\rho\left(\mu, \varphi, \mu^{\prime}, \varphi^{\prime}\right) \quad 0 \quad 0 \quad 0\right]$,

where $\rho\left(\mu, \varphi, \mu^{\prime}, \varphi^{\prime}\right)$ is the scalar bidirectional reflectance distribution function (BRDF). Currently, the APC code includes three types of widely used BRDF models for land: 1 - Lambertian surface; 2a - RPV model [50]; 2b - modified RPV (MRPV) model [39] 3 - Ross Thick - Li Sparse (RTLS) model [35]. The computational subroutines were adapted from the code SHARM (see Eqs. (A11)-(A14) and (A15)-(A19) from Lyapustin [36]). Eq. (41) is expanded into Fourier series

$\rho\left(\mu, \mu^{\prime}, \varphi-\varphi^{\prime}\right)=\left(2-\delta_{0, m}\right) \sum_{m=0}^{M} \rho^{m}\left(\mu, \mu^{\prime}\right) \cos \left(m\left(\varphi-\varphi^{\prime}\right)\right)$,

where

$\rho^{m}\left(\mu, \mu^{\prime}\right)=\frac{1}{2 \pi} \int_{0}^{2 \pi} \rho\left(\mu, \mu^{\prime}, \varphi\right) \cos (m \varphi) d \varphi$.

Eq. (43) is integrated numerically using the Double Gauss scheme.

Along with the scalar BRDF, code APC includes the BPDF model for a wind-ruffled ocean surface (see e.g., [34, Section 5]). In this case, the BPDF is

$\mathbf{R}\left(\mu, \mu^{\prime}, \varphi-\varphi^{\prime}\right)=S\left(\mu, \mu^{\prime}, u, \phi\right) \mathbf{M}\left(\mu, \mu^{\prime}, \varphi-\varphi^{\prime}\right)$.

In Eq. (44) $S\left(\mu, \mu^{\prime}, u, \phi\right)$ is the bidirectional shadowing function that includes mutual shadowing of waves and surface roughness in terms of the probability distribution function of the slopes. The roughness of the surface depends on the near-surface wind speed, $u$, for the azimuthally independent model by Nakajima and Tanaka [43]. The azimuthally dependent Gram-Charlier model [11] includes the direction of the wind, $\phi$. The algorithm of computation of the function $S\left(\mu, \mu^{\prime}, u, \phi\right)$ is provided in literature (e.g., [36, Appendices $C$ and $D$ ] for the azimuthally independent and azimuthally depended cases respectively).

The second factor in Eq. (44), $\mathbf{M}\left(\mu, \mu^{\prime}, \varphi-\varphi^{\prime}\right)$, is the matrix that includes polarization effects. It contains the known Fresnel reflection matrix [47, Eq. (A.1)]. This matrix is defined with respect to the plane of incidence and reflection of a beam. In general, this plane does not coincide with the plane defining VRTE solution. In order to match these two planes, the rotator is applied to the Fresnel matrix. This is similar to the scattering process described by the scattering integral in Eq. (2).

Two methods of computation of $\mathbf{M}\left(\mu, \mu^{\prime}, \varphi-\varphi^{\prime}\right)$ are known. The first one is described by Eqs. (64)-(79) from Mishchenko and Travis [41] and uses the amplitude matrix that transforms the components of the electro-magnetic 
field. In this case, the law of reflection of the parallel and perpendicular components of the electro-magnetic field from a dielectric surface (Fresnel formulas) must be known. Hence, this approach is very convenient for the ocean surface.

Unfortunately, this is generally not the case for most natural surfaces including soil, vegetation etc. The Mueller matrices of the surface are often measured experimentally [48]. In these cases, it is necessary to perform the rotation of the Mueller matrix explicitly using the rotation matrix [1, Eq. (A4)]. This approach does not require the law of reflection of the electro-magnetic field, but the angles of rotation must be computed explicitly.

In order to do this, one has to take into account that the Mueller matrix of the surface, including the rotators, is written with respect to the surface normal [41] which is the opposite to the direction of the positive cosines of the VRTE, Eq. (2). For the surface reflection, both incident and reflected angles are often assumed to be within $[0,90)$ degrees [50] which is also different from the definition of angles used in the VRTE.

In order to avoid the above mentioned mismatches, the explicit relation for $\mathbf{M}\left(\mu, \mu^{\prime}, \varphi-\varphi^{\prime}\right)$ is provided in this paper. He et al. [19] have provided the reflection-transmission Mueller matrices of the facet (II 2.4.1 and 2.4.2 of their paper), but they did not explicitly discuss the problem of Fourier expansion of the Mueller matrix. The process of reflection is considered similarly to the process of scattering with the obvious exception, that for reflection the directions of incidence and "scattering" always belong to the same hemisphere. In order to match the coordinate systems of the VRTE and the reflecting surface, we use one normal direction as defined in Section 1. It means that the signs of the angles of rotation during reflection are equal to those during scattering (see Eq. (11))

$\mathbf{M}\left(\mu, \mu^{\prime}, \varphi-\varphi^{\prime}\right)=\mathbf{L}\left(\pi-\sigma_{2}\right) \mathbf{F}_{R}(\theta) \mathbf{L}\left(-\sigma_{1}\right)$,

where $\theta$ is a half of an angle complementary to the "scattering" angle Eq. (13). The elements of the Fresnel matrix

$\mathbf{F}_{R}(\theta)=\left[\begin{array}{cccc}r_{+} & r_{-} & 0 & 0 \\ r_{-} & r_{+} & 0 & 0 \\ 0 & 0 & r_{0} & 0 \\ 0 & 0 & 0 & r_{0}\end{array}\right]$

are known [47, Eqs. (A1)-(A3)]. Using the notation

$c_{1,2}=\cos \left(2 \sigma_{1,2}\right) ; \quad s_{1,2}=\sin \left(2 \sigma_{1,2}\right)$,

and Eq. (45), one can write

$\mathbf{M}\left(\mu, \mu^{\prime}, \varphi-\varphi^{\prime}\right)=\left[\begin{array}{cccc}r_{+} & r_{-} c_{1} & -r_{-} s_{1} & 0 \\ r_{-} c_{2} & r_{+} c_{1} c_{2}-r_{0} s_{1} s_{2} & -r_{+} s_{1} c_{2}-r_{0} c_{1} s_{2} & 0 \\ r_{-} s_{2} & r_{+} c_{1} s_{2}+r_{0} s_{1} c_{2} & -r_{+} s_{1} s_{2}+r_{0} c_{1} c_{2} & 0 \\ 0 & 0 & 0 & r_{0}\end{array}\right]$.

Further on, the following definition $\{\mu<0, \varphi\}$ and $\left\{\mu^{\prime}>0, \varphi^{\prime}\right\}$ is used for the direction of reflection (observation) and incidence, respectively. The angle $2 \theta$ is

$\cos 2 \theta=\cos (\pi-\Theta)=-\mu \mu^{\prime}-\sqrt{1-\mu^{2}} \sqrt{1-\mu^{\prime 2}} \cos \varphi$.
Eq. (49) allows to compute the elements of $\mathbf{F}_{R}(\theta)$ in Eq. (46). The angles in Eq. (47) are computed using Eqs. (3.11), (3.12) and (3.23) from TPL without any modifications. Here the particular attention is paid only to Eq. (3.23) from TPL

$\frac{\sin \sigma_{1}}{\sqrt{1-\mu^{2}}}=\frac{\sin \sigma_{2}}{\sqrt{1-\mu^{\prime 2}}}=-\frac{\sin \left(\varphi-\varphi^{\prime}\right)}{\sqrt{1-\cos ^{2} \Theta}}$

where L'Hopital's rule is used in Eq. (49) for $|\mu|=1$ or $\left|\mu^{\prime}\right|=1$. Finally, no rotation is performed for $\varphi-\varphi^{\prime}=0, \pi$

(TPL, p. 71).

The azimuthal expansion of Eq. (48) is similar to the azimuthal expansion of the matrix Eq. (11). Using Eq. (18) from Siewert [55] and the definition

$C_{m}=\cos (m \varphi) ; S_{m}=\sin (m \varphi)$,

one can write

$$
\begin{aligned}
\mathbf{M}\left(\mu, \mu^{\prime}, \varphi-\varphi^{\prime}\right) & =\sum_{m=0}^{M}\left(2-\delta_{0, m}\right)\left[\begin{array}{cccc}
C_{m} M_{11}^{m} & C_{m} M_{21}^{m} & -S_{m} M_{13}^{m} & 0 \\
C_{m} M_{21}^{m} & C_{m} M_{22}^{m} & -S_{m} M_{23}^{m} & 0 \\
S_{m} M_{31}^{m} & S_{m} M_{32}^{m} & C_{m} M_{33}^{m} & 0 \\
0 & 0 & 0 & C_{m} M_{44}^{m}
\end{array}\right] \\
& =\sum_{m=0}^{M}\left(2-\delta_{0, m}\right) \mathbf{M}^{m}\left(\mu, \mu^{\prime}, \varphi-\varphi^{\prime}\right) .
\end{aligned}
$$

Eqs. (48) and (52) provides the law of Fourier expansion of the elements of the Mueller matrix. For example, $M_{11}^{m}$ is computed using Eq. (42). Lenoble et al. [34, Sections 5.2.1 and 5.2.2] provided detailed analysis of Eqs. (44)-(48). Similar to the phase matrix, they used analytical integration over azimuth to get the Fourier expansion moments $\mathbf{M}^{m}\left(\mu, \mu^{\prime}, \varphi-\varphi^{\prime}\right)$. This approach is more complicated than the numeric integration over azimuth and still requires numeric integration over zenith angle to compute expansion moments ([34, Eq. (45)]).

To conclude this section, we note that APC uses the postprocessing correction of the direct beam reflected from the surface and transmitted to the TOA. The correction term is expressed as

$$
\begin{aligned}
\overrightarrow{\boldsymbol{\Delta}}_{\mathrm{TOA}}\left(\mu_{-}, \varphi\right)= & \frac{\mu_{0}}{\pi}\left[\mathbf{R}\left(\mu_{-}, \varphi, \mu_{0}, \varphi_{0}\right)-\sum_{m=0}^{M}\left(2-\delta_{0, m}\right) \mathbf{R}^{m}\left(\mu_{-}, \varphi, \mu_{0}, \varphi_{0}\right)\right] \\
& \times \exp \left(\frac{\mu_{0}-\mu_{-}}{\mu_{0} \mu_{-}}\right) \overrightarrow{\mathbf{S}}_{0}
\end{aligned}
$$

where $\mathbf{R}^{m}\left(\mu_{-}, \varphi, \mu_{0}, \varphi_{0}\right)$ is defined by Eq. (52). The correction term, $\overrightarrow{\boldsymbol{\Delta}}_{T O A}$, is applied to the resulting Stokes vector at the TOA

$$
\overrightarrow{\mathbf{S}}_{\mathrm{TOA}}\left(\tau=0, \mu_{-}, \varphi\right)=\overrightarrow{\mathbf{S}}\left(\tau=0, \mu_{-}, \varphi\right)+\overrightarrow{\boldsymbol{\Delta}}_{\mathrm{TOA}}\left(\mu_{-}, \varphi\right)
$$

and does not affect transmission of radiation through the atmosphere. Eq. (53) exactly describes the surface signal, transmitted through the atmosphere without scattering. The forward peak is included in the small-angle part and does not require correction.

This section provided the detailed formulation for the surface reflection. The algorithm of computation of the Mueller matrix that includes the explicit rotation of the reference plane is described in the coordinate system of the VRTE Eq. (2). All necessary expressions are provided either explicitly or by means of the direct reference to the 

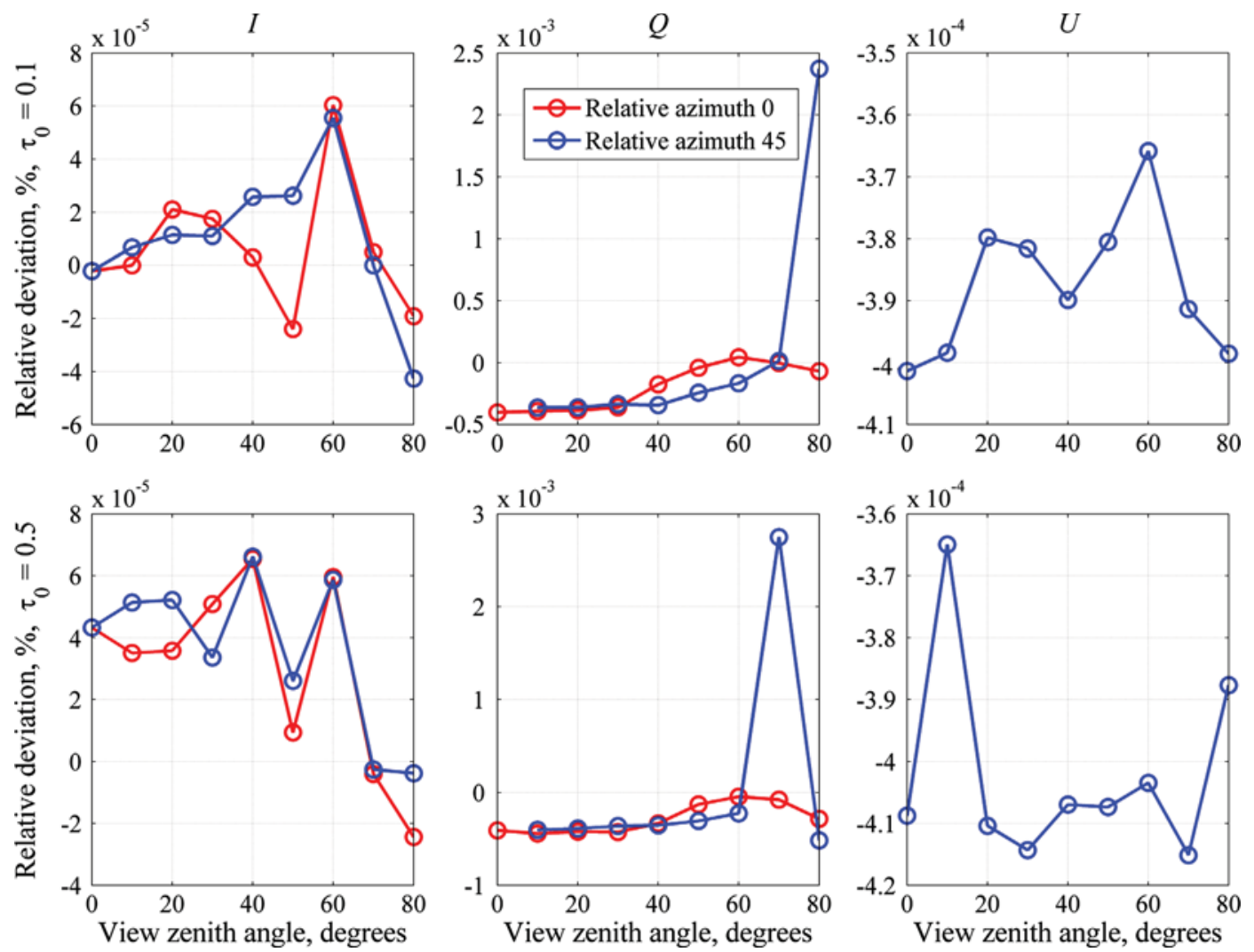

Fig. 2. The relative deviation, \%, between APC and SCIATRAN for $I$ (left column), $Q$ (middle column), and $U$ (right column) components of the Stokes vector. Solar zenith angle $60^{\circ}$, relative azimuth $0^{\circ}$ (red lines) and $45^{\circ}$ (blue lines). View zenith angles from $0^{\circ}$ to $80^{\circ}$, with $10^{\circ}$ step (reflected radiation). Rayleigh atmosphere with optical thickness 0.1 (top row) and 0.5(bottom tow). Single scattering albedo 0.9, no depolarization. Ruffled ocean surface was simulated using Nakajima-Tanaka model. Wind speed $1 \mathrm{~m} / \mathrm{s}$ at $10 \mathrm{~m}$ above the surface. (For interpretation of the references to color in this figure legend, the reader is referred to the web version of this article.)

published equations. This concludes the description of the theoretical background of the APC code. In the last Section 6 some numerical results are provided.

\section{Numerical results}

The code APC, written in FORTRAN, was thoroughly tested against its Matlab prototype, MVDOM, which in turn was validated against SCIATRAN, Pstar, and other codes involved in the benchmark test described in Kokhanovsky et al. [28]. For a homogeneous and vertically stratified media and for black and Lambertian reflecting surfaces the code was tested in Korkin et al. [29] showing good (five significant digits) agreement with RT3 [13]. Code SHARM [36] was used to validate the scalar surface BRDF models adapted in code APC.

To test the ocean BPDF model given by Eqs. (43) and (48), and atmosphere-ocean interaction, we made a direct comparison with code SCIATRAN [53]. The SCIATRAN software package along with a detailed User's Guide is available via the webpage of the Institute of Environmental Physics (IUP), University of Bremen: http://www.iup.phy sik.uni-bremen.de/sciatran. As an example, we present computations of the reflected radiation over the ocean in Rayleigh case for the geometry $\theta_{o}=60^{\circ}$, relative azimuths $\varphi=0$ (dash lines) and $45^{\circ}$ (solid lines), and the VZA from
0 to 80 with 10 degrees step. The following atmospheric parameters (OD $\tau=0.1$, and 0.5 , SSA $\omega_{0}=0.9$, and zero depolarization factor) and the wind speed $1 \mathrm{~m} / \mathrm{s}$ with Nakajima and Tanaka [44] model were used. The relative difference between the APC and SCIATRAN results for $I, Q$, and $U$ (for nonzero azimuth only) is shown in Fig. 2. The relative deviation between the two codes was found to be negligible.

To illustrate functionality of the developed code, the next example shows the sensitivity of the intensity, $I$, and the degree of polarization

$p=\sqrt{Q^{2}+U^{2}+V^{2}} / I$

to the shape of particles represented by spheres and spheroids. The simulations were performed for an idealized two-layer atmosphere illuminated at $\mathrm{SZA}=45^{\circ}$. The top layer is assumed to be purely Rayleigh with optical depth $\tau_{R}=0.3$, and the bottom layer consisting of the nonabsorbing aerosol only. The surface bidirectional reflectance was simulated using RPV model with parameters typical of savannah [36]. The aerosols are represented by spherical particles and spheroids with integrated aspect ratio close to 3. Fig. 3 shows the phase matrices for spheres and spheroids, respectively. Note, that all diagonal elements, $a_{1,2,3,4}$, of the scattering matrix contain scattering 

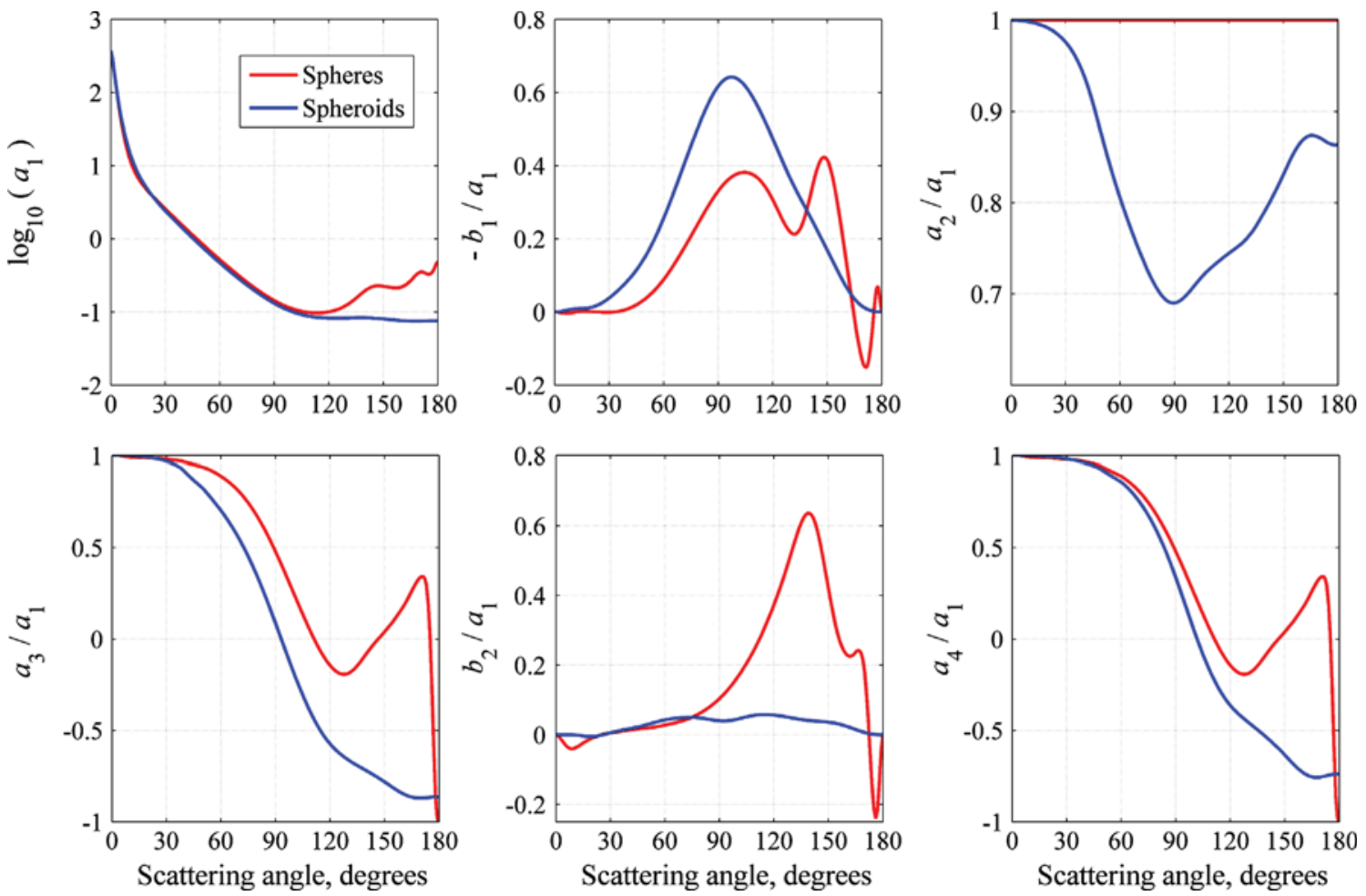

Fig. 3. The elements of the phase matrices for spheres (red line) and spheroids (blue line) used for computation. See details in Section 6. (For interpretation of the references to color in this figure legend, the reader is referred to the web version of this article.)

anisotropy. Computations were performed for two values of aerosol optical depth, $\tau_{A}=0.1,0.5$ and the geometry of $\mathrm{VZA}=180^{\circ}, 150^{\circ}, 120^{\circ}$ (transmitted radiation), and $0^{\circ}, 30^{\circ}$, $60^{\circ}$ (reflected radiation) for the full range of azimuthal angles with $5^{\circ}$ step. The blue, green and red colors represent different $\mathrm{VZA}$ (e.g., to $\mathrm{VZA}=0^{\circ}, 30^{\circ}, 60^{\circ}$ for the case of reflection).

The results for the total intensity, $I$, and for the degree of polarization, $P$ (see Eq. (55)), are shown in Fig. 4 for both reflected and transmitted radiation. The solid and dashed lines represent spherical aerosols and spheroids, respectively. In the considered case, the figures clearly show that polarization is more sensitive to the shape of the particles even if depolarizing surface is assumed. In case of nadir observation, sensitivity of the total intensity to the particles' shape is negligible (Fig. 4a and e) whereas sensitivity of polarization remains considerable (Fig. $4 \mathrm{~b}$ and f). The same holds true for the transmitted radiation.

\section{Conclusion}

In this paper, we presented a new code, APC, that uses the decomposition of the diffuse light field into a regular and anisotropic parts. Contrary to approximations that truncate the phase matrix, this approach does not modify the scattering law. As in the scalar case, the regular part of the signal, which requires the numerical solution, is smoothed in both zenith and azimuth angles after subtraction of the anisotropic component. The anisotropic part is computed analytically, while the discrete ordinates method is used for numerical computation of the regular part. The code is developed in Fortran 90/95 and uses
LAPACK package to perform the singular value decomposition and matrix operations.

For the users' convenience, the APC code has built-in atmospheric profiles and several common models of the surface bidirectional reflectance. This paper paid a particular attention to the problem of reflection of polarized light from the waved ocean surface. We have provided the explicit formulas and direct references to the equations that describe the reflection of polarized light from any surface, for which the Mueller matrix is known, while the law of reflection of the components of the electro-magnetic field may be unknown. Although the equations are not new, we are not aware of the detailed description of the complete model provided here. In our approach, an attempt was made to keep the same coordinate system for the VRTE problem and the boundary condition. The code is available at ftp://climate1.gsfc.nasa.gov/skorkin. The kernel databases for fast spheric and spheroidal scattering computations are also provided by courtesy of Dubovik [12].

\section{Acknowledgments}

The first author thanks O. Dubovik and T. Lapyonok (Laboratory of Atmospheric Optics, Lille, France) for the spheres/spheroids package, and D.S. Efremenko (Remote Sensing Technology Institute, German Aerospace Center) for valuable discussion of the numerical methods in the radiative transfer problems and their implementation in modern Fortran. 
a

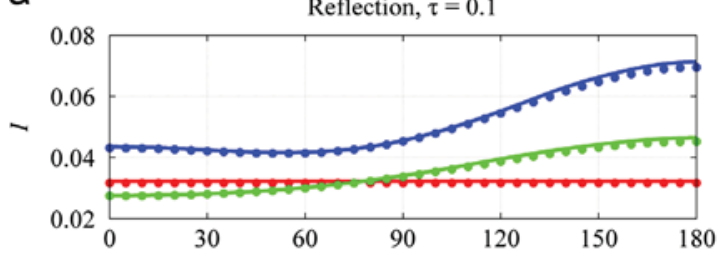

C

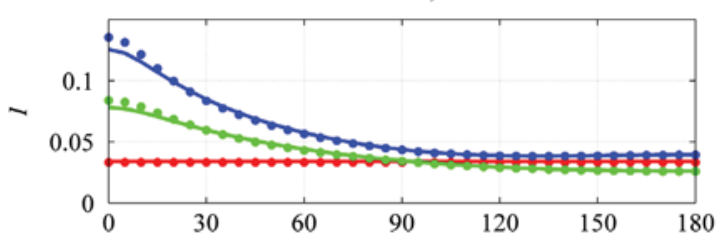

e

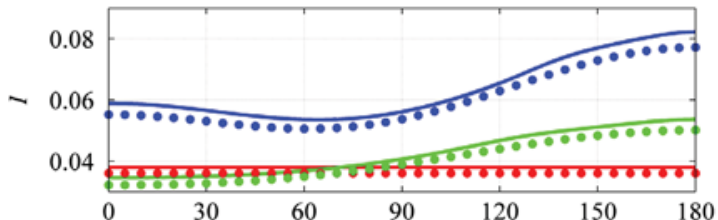

g

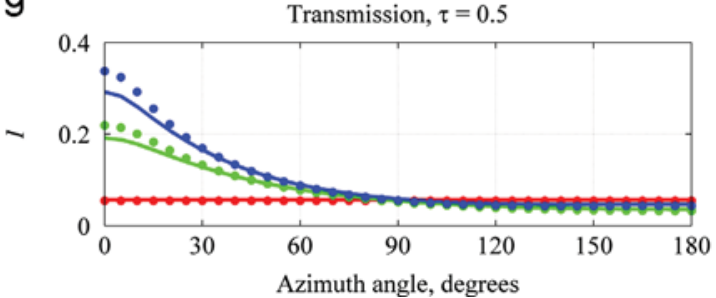

b

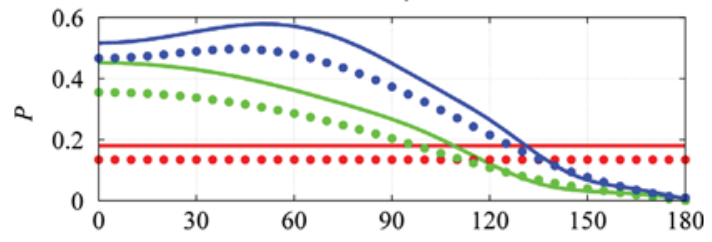

d

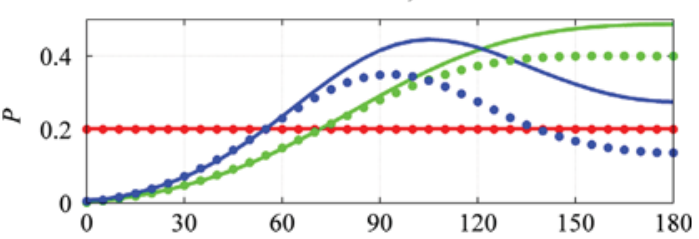

f

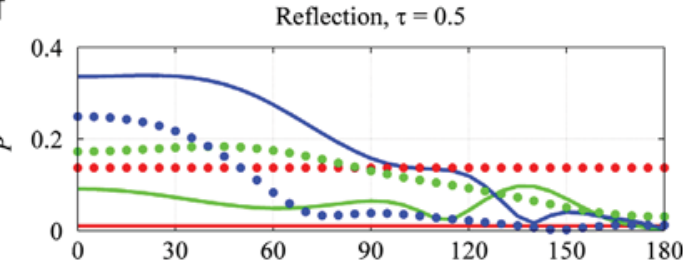

h

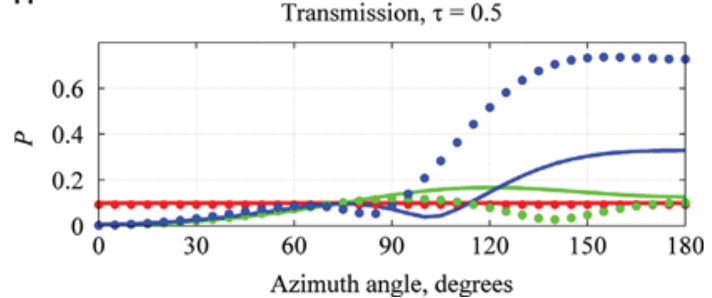

Fig. 4. The values of the total intensity, $I$, and the degree of polarization, $P$, in case of spheres (solid lines) and spheroids (dots) as functions of the relative azimuth. $\mathrm{VZA}=180^{\circ}$ (red), $150^{\circ}$ (green), $120^{\circ}$ (blue) for the transmitted light (c, d, g, h) and $\mathrm{VZA}=0^{\circ}$ (red), $30^{\circ}$ (green), $60^{\circ}$ (blue) for the reflected light (a, b, $\mathrm{e}, \mathrm{f}$ ). The results are shown for two different optical depths of the aerosol layer. (For interpretation of the references to color in this figure legend, the reader is referred to the web version of this article.)

\section{References}

[1] Ahmad Z, Fraser RS. An iterative transfer code for ocean-atmosphere systems. J Atmos Sci 1982;39:656-65.

[2] Astakhov IE, Budak VP, Lisitsin DV, Selivanov VA. Solution of the vector radiative transfer equation in the small angle approximation of the spherical harmonics method. Atmos Oceanic Opt 1994;7:398-403.

[3] Budak VP, Sarmin SE. Solution of the radiative transfer equation by the method of spherical harmonics in the small-angle modification. Atmos Oceanic Opt 1990;3:898-903.

[4] Budak VP, Korkin SV. The spatial polarization distribution over the dome if the sky for abnormal irradiance of the atmosphere. J Quant Spectrosc Radiat Transfer 2008;109:1347-62.

[5] Budak VP, Korkin SV. The method of subtraction of the anisotropic part of the light field from solution of the vector radiative transfer equation. Bull Moscow Power-Eng Inst 2008;5:120-6 [in Russian].

[6] Budak VP, Klyuykov DA, Korkin SV. Convergence acceleration of radiative transfer equation solution at strongly anisotropic scattering. In: Kokhanovsky AA, editor. Light scattering reviews 5. Single light scattering and radiative transfer. Chichester: Springer; 2010. p. 147-204.

[7] Benassi M, Garcia RDM, Siewert CE. A generalized spherical harmonics solution basic to the scattering of polarized light. Z Angew Math Phys 1985;36:70-88.
[8] Chalhoub ES, Garcia RDM. The equivalence between two techniques of angular interpolation for the discrete-ordinates method. J Quant Spectrosc Radiat Transfer 2000;64:517-35.

[9] Chandrasekhar S. Radiative transfer. London: Oxford University Press; 1950.

[10] Compiègne $\mathrm{M}$, C-Labonnote $\mathrm{L}$, Dubuisson $\mathrm{P}$. The phase matrix truncation impact on polarized radiance. In: AIP conference proceedings of the radiarion processes in the atmosphere and ocean, IRS2012, vol. 1531; 2013. p. 95-8.

[11] Cox C, Munk W. Measurements of the roughness of the sea surface from photographs of the Sun's glitter. J Opt Soc Am 1954;44:838-50.

[12] Dubovik O, Sinyuk A, Lapyonok T, Holben BN, Mishchenko M, Yang P, et al. Application of light scattering by spheroids for accounting for particle non-sphericity in remote sensing of desert dust. Geophys Res Lett 2006;111:D11208. http://dx.doi.org/10.1029/2005JD006619d.

[13] Evans KF, Stephens GL. A new polarized atmospheric radiative transfer model. J Quant Spectrosc Radiat Transfer 1991;46(5): 413-23.

[14] Garcia RDM, Siewert CE. A generalized spherical harmonics solution for radiative transfer models that include polarization effects. J Quant Spectrosc Radiat Transfer 1986;36(5):401-23.

[15] Garcia RDM, Siewert CE. A simplified implementation of the discrete-ordinates method for a class of problems in radiative transfer with polarization. J Quant Spectrosc Radiat Transfer 2011;112:2801-13. 
[16] Gelfand IM, Minlos RA, Shapiro ZYa. Representations of the rotation and Lorentz groups and their applications. Oxford: Pergamon Press; 1963.

[17] Goudsmit S, Saunderson JL. Multiple scattering of electrons. Phys Rev 1940;57:24-9.

[18] Hansen JE, Travis LD. Light scattering in planetary atmospheres. Space Sci Rev 1974;16:265-81.

[19] He X, Bai Y, Zhu Q Gong F. A vector radiative transfer model of coupled ocean-atmosphere system using matrix-operator method for rough sea-surface. J Quant Spectrosc Radiat Transfer 2010;111: 1426-48.

[20] Hovenier JW. Multiple scattering of polarized light in planetary atmospheres. Astron Astrophys 1971;13:7-29.

[21] Hovenier JW, van der Mee C. Fundamental relationships relevant to the transfer of polarized light in a scattering atmosphere. Astron Astrophys 1983;128:1-16.

[22] Hovenier JW, van der Mee C, Domke H. Transfer of polarized light in planetary atmocpheres. Basic concepts and practical methods. Dordrecht: Kluwer Academic Publisher; 2004.

[23] van de Hulst HC. Light scattering by small particles. New York: Wiley; 1957.

[24] Irvine WM. Diffuse reflection and transmission by cloud and dust layers. J Quant Spectrosc Radiat Transfer 1968;8:471-85.

[25] Karp AH, Greenstadt J, Fillmore JA. Radiative transfer through an arbitrary thick scattering atmosphere. J Quant Spectrosc Radiat Transfer 1980;24:391-406.

[26] Kattawar GW, Plass GN. Radiance and polarization of multiple scattered light from haze and clouds. Appl Opt 1968;7(N8):1519-27.

[27] Kneizys FX, Abreu LW, Anderson GP, Chetwynd JH, Shettle EP, Berk A, et al. The MODTRAN 2/3 report and LOWTRAN 7 model. North Andover, MA: Ontar Corporation; 1996.

[28] Kokhanovsky AA, Budak VP, Cornet C, Duan M, Emde C, Katsev IL, et al. Benchmark results in vector atmospheric radiative transfer. J Quant Spectrosc Radiat Transfer 2010;111:1931-46.

[29] Korkin SV, Budak VP, Lyapustin AI. Modified vector discrete ordinates method for radiative transfer in vertically inhomogeneous atmospheres. In: Mishchenko M, Yatskiv Ya, Rosenbush V, Videen G, editors. Proceedings of the NATO ASI on "special detection technique (Polarimetry) and reomote sensing”, 12-25 September, 2010. Kyiv, Ukraine; 2010. p. 76.

[30] Korkin SV, Lyapustin AI, Rozanov VV. Analysis of the radiative transfer equation with highly asymmetric phase function. J Quant Spectrosc Radiat Transfer 2011;112:1595-608.

[31] Korkin SV, Lyapustin AI, Rozanov VV. Modifications of discrete ordinate method for computations with high scattering anisotropy: comparative analysis. J Quant Spectrosc Radiat Transfer 2012;113: 2040-8.

[32] Kuščer I, Ribarič M. Matrix formalism in the theory of diffusion of light. Opt Acta 1959;6:42-51.

[33] Lenoble J, editor. Radiative transfer in scattering and absorbing atmospheres: standard computational procedures. Hampton, Virginia, USA: A. Deepak Publishing; 1985.

[34] Lenoble J, Herman M, Deuzé JL, Lafrance B, Santer R, Tanré D. A successive order of scattering code for solving the vector equation of transfer in the earth's atmosphere with aerosols. J Quant Spectrosc Radiat Transfer 2007; 107:479-507.

[35] Lucht W, Schaaf CB, Strahler AH. An algorithm for the retrieval of albedo from space using semiempirical BRDF models. IEEE Trans Geosci Remote Sens Soc 2000;38:977-98.

[36] Lyapustin A. Radiative transfer code SHARM for atmospheric and terrestrial applications. Appl Opt 2005;44(3):7764-72.

[37] Marchuk GI, Mikhailov GA, Nazaraliev MA. The Monte-Carlo methods in atmospheric optics. Berlin: Springer; 1980.

[38] Mark C. The neutron density near a plane surface. Phys Rev 1947;72: 558-64.

[39] Martonchik JV, Diner DJ, Pinty B, Verstratete MM, Myneni RB, Yu Knyazikhin, et al. Determination of land and ocean reflective, radiative and biophysical properties using multiangle imaging. IEEE Trans Geosci Remote Sens Soc 1998;36:1266-81.

[40] Mishchenko MI. The fast invariant embedding method for polarized light: computational aspects and numerical results for Rayleigh scattering. J Quant Spectrosc Radiat Transfer 1990;43(N2):163-71.

[41] Mishchenko MI, Travis LD. Satellite retrieval of aerosol properties over ocean using polarization as well as intensity of reflected sunlight. J Geophys Res 1997;102(D14):16989-7013.

[42] Mayer B. Radiative transfer in the cloudy atmosphere. Eur Phys J Conf 2009;1:75-99.

[43] Nakajima T, Tanaka M. Effect of wind-generated waves on the transfer of solar radiation in the atmosphere-ocean system. J Quant Spectrosc Radiat Transfer 1983;29:521-37.

[44] Nakajima T, Tanaka M. Matrix formulations for the transfer of solar radiation in a plane-parallel scattering atmosphere. J Quant Spectrosc Radiat Transfer 1986;35:13-21.

[45] Nakajima T, Tanaka M. Algorithms for radiative intensity calculations in moderately thick atmospheres using a truncation approximation. J Quant Spectrosc Radiat Transfer 1988;40(N1):51-69.

[46] Natraj V, Spurr RJD. A fast pseudo-spherical two orders of scattering model to account for polarization in vertically inhomogeneous scattering-absorbing media. J Quant Spectrosc Radiat Transfer 2007:107:263-93.

[47] Ota Y, Higurashi A, Nakajima T, Yokota T. Matrix formulations of radiative transfer including the polarization effect in a coupled atmosphere-ocean system. I Quant Spectrosc Radiat Transfer 2010;111:878-94.

[48] Peltoniemi JI, Hakala T, Suomalainen J, Puttonen E. Polarised bidirectional reflectance factor measurement from soil, stones, and snow. J Quant Spectrosc Radiat Transfer 2009;110:1940-53.

[49] Plass GN, Kattawar GW, Catchings FE. Matrix operator theory of radiative transfer. 1: Rayleigh scattering. Appl Opt 1973;12:314-29.

[50] Rahman H, Pinty B, Verstraete MM. Coupled surface-atmosphere reflectance (CSAR) model. 2. Semiempirical surface model usable with NOAA advanced very high resolution radiometer data. J Geophys Res 1993;98:20,791-801.

[51] Romanova LM. Solution of the radiative transfer equation in the case of scattering phase function greatly differs from a spherical one. Opt Spectrosc 1962;13:429-35 (in Russian).

[52] Rozanov A, Rozanov V, Buchwitz M, Kokhanovsky A, Burrows JP. SCIATRAN 2.0-a new radiative transfer model for geophysical applications in the $175-2400 \mathrm{~nm}$ spectral range. Adv Space Res 2005;36(N5):1015-9.

[53] Rozanov VV, Rozanov AV, Kokhanovsky AA, Burrows JP. Radiative transfer through terrestrial atmosphere and ocean: software package SCIATRAN. J Quant Spectrosc Radiat Transfer 2013 http://dx.doi. org/10.1016/j.jqsrt.2013.07.004.

[54] Siewert CE. On the phase matrix basic to the scattering of polarized light. Astron Astrophys 1982;109:195-200.

[55] Siewert CE. A discrete-ordinates solution for radiative-transfer models that include polarization effects. J Quant Spectrosc Radiat Transfer 2000;64:227-54.

[56] Spurr RJD. VLIDORT: linearized pseudo-spherical vector discrete ordinate radiative transfer code for forward model and retrieval studies in multilayer multiple scattering media. J Quant Spectrosc Radiat Transfer 2006;102(N2):316-42.

[57] Sykes J. Approximate integration of the equation of transfer. Mon Not R Astron Soc 1951;11:377-86.

[58] Thomas GE, Stamnes K. Radiative transfer in atmosphere and ocean. Cambridge: University Press; 1999

[59] Ustinov EA. Method of spherical harmonics: application to the transfer of polarized radiation in a vertically non-uniform planetary atmosphere. Mathematical apparatus. Cosmic Res 1988;26:473-84.

[60] Wiscombe WJ. The delta-M method: rapid yet accurate radiative flux calculations for strongly asymmetric phase functions. J Atmos Sci 1977; 34:1408-22.

[61] Zwillinger D, editor. CRC standard mathematical tables and formulae. Boca Raton, FL: CRC Press; 1995. 